\section{Ethephon Substrate Drenches Inhibit Stem Extension of Floriculture Crops}

\author{
William B. Miller ${ }^{3}$, Neil S. Mattson, Xiaorong Xie ${ }^{1}$, and Danghui $\mathrm{Xu}^{2}$ \\ Department of Horticulture, Cornell University, 134A Plant Science Building, \\ Ithaca, NY 14853 \\ Christopher J. Currey, Kasey L. Clemens, and Roberto G. Lopez \\ Department of Horticulture and Landscape Architecture, 625 Agriculture \\ Mall Drive, West Lafayette, IN 47907
}

Michael Olrich and Erik S. Runkle

Department of Horticulture, Michigan State University, A288 PSSB, East Lansing, MI 48824

Additional index words. bedding plants, Dutch bulbs, ethylene, plant growth regulators

\begin{abstract}
Ethephon [(2-chloroethyl) phosphonic acid] is a plant growth regulator that releases ethylene on application and can abort flowers, stimulate branching, and inhibit stem elongation. Although ethephon is used as a foliar spray during the commercial production of many ornamental crops, its effectiveness as a drench has not been widely investigated. We performed experiments to quantify the effects of an ethephon drench on growth and flowering of a range of bedding plant and Narcissus cultivars and to assess the effect of lime on ethylene release from a peat substrate. A substrate drench of 0,100 , 250 , or $500 \mathrm{mg} \cdot \mathrm{L}^{-1}$ ethephon was applied to 12 potted Narcissus cultivars at one location, and up to $200 \mathrm{mg} \cdot \mathrm{L}^{-1}$ was applied to 24 cultivars of bedding plants at three locations. Compared with untreated controls, ethephon generally reduced plant height at flowering and the effect increased with increased concentration. For example, Narcissus treated with a $250 \mathrm{mg} \cdot \mathrm{L}^{-1}$ ethephon drench had stems that were $20 \%$ to $40 \%$ shorter at the end of flowering than control plants. However, ethephon drenches generally caused a 2- to 3-day flowering delay, and two cultivars had a phytotoxic response. Among the bedding plants studied, a 100-mg. $\mathrm{L}^{-1}$ ethephon drench suppressed plant height at flowering by greater than $30 \%$ in Catharanthus, Celosia, Dianthus, and Verbena, but by only $10 \%$ to 15\% in Lobelia, Lycopersicon, and Tagetes. The drenches also delayed flowering in 10 of the $\mathbf{1 6}$ crops measured and decreased dry mass accumulation in all of the crops measured. Ethephon release from peat substrate became maximal $\approx 120 \mathrm{~h}$ after application and was dramatically increased by incorporation of dolomitic lime up to a rate of $9.5 \mathrm{~kg}$ lime per $\mathrm{m}^{3}$ of peat. Collectively, these studies show that ethephon substrate drenches inhibit stem elongation in a broad range of floriculture crops, but can also delay flowering and reduce biomass accumulation.
\end{abstract}

Potted spring-flowering bulbs and bedding and garden plants collectively account for $\$ 1.97$ billion $(49 \%)$ of the total U.S. wholesale value of floriculture crops for the 15 top-producing states (U.S. Department of Agriculture, 2011). These crops often have excessive stem elongation as a result of dense spacing or suboptimal environmental conditions, which results in overgrown, unattractive, and unmarketable plants (Krug et al., 2006a,b; Starman et al., 2004). For many potted flowering plants such as Euphorbia pulcherrima Willd. ex Klotzsch, Lilium longiflorum Thunb. and Lilium L. hybrids, and Narcissus pseudonarcissus L., there are industry target height

Received for publication 12 May 2012. Accepted for publication 30 July 2012.

${ }^{1}$ Department of Biology, Hexi University, Zhangye, China.

${ }^{2}$ School of Life Science, Lanzhou University, Lanzhou, China.

${ }^{3}$ To whom reprint requests should be addressed; e-mailwbm8@cornell.edu.
Rademacher, 2000). In contrast, ethephon [(2-chloroethyl) phosphonic acid] is a PGR that releases ethylene $\left(\mathrm{C}_{2} \mathrm{H}_{4}\right)$, chlorine $\left(\mathrm{Cl}^{-}\right)$, and hydrogen phosphate $\left(\mathrm{H}_{2} \mathrm{PO}_{4}^{-}\right)$on application and is known to inhibit internode elongation, induce branching, and cause abscission of flower buds and leaves (Glady et al., 2007; Leatherwood et al., 2009; Maynard and Swan, 1963; Starman et al., 2004).

Ethephon sprays are applied to many floriculture crops 1 to 2 weeks after transplant and at 1- to 2-week intervals thereafter (Styer, 2002). Application concentrations for annual and perennials generally range from 250 to $1000 \mathrm{mg} \cdot \mathrm{L}^{-1}$ (Glady et al., 2007; Starman et al., 2004; Styer, 2002). Currently, ethephon sprays are the main technique for height control in Hyacinthus orientalis L. and Narcissus with suggested concentrations of 500 to $2000 \mathrm{mg} \cdot \mathrm{L}^{-1}$ depending on the cultivar, length of cooling, and forcing time (De Hertogh, 1996).

Substrate drenches of many PGRs provide more uniform results and increase the duration of effectiveness compared with foliar sprays (Boldt, 2008; Gent and McAvoy, 2000). Applying antigibberellin substrate drenches containing flurprimidol, paclobutrazol, or uniconazole early in the production of Euphorbia, Lilium longiflorum, and bedding plants is a PGR application strategy for height control (Barrett, 2004; Currey et al., 2010; Currey and Lopez, 2011; Lopez and Runkle, 2007; Runkle et al., 2006).

Although ethephon sprays are effective at inhibiting stem elongation and promoting branching, the potential of ethephon substrate drenches for bedding plant and spring bulb stem elongation control is largely unknown. Johnson et al. (1982) demonstrated growth effects from soil-applied ethephon on Ficus benjamina and preliminary results on Narcissus height control from substrate applications of ethephon have been reported (Anonymous, 1973, 1974; Briggs, 1975). However, to our knowledge, no literature on the use of ethephon as a substrate drench on bedding plants exists. Therefore, the objectives of this research were to evaluate ethephon drenches on a range of bedding plant and Narcissus cultivars and to assess the effect of substrate lime concentrations on ethylene release from a peat substrate.

\section{Materials and Methods}

Expt. 1: Ethephon drenches on Narcissus at Cornell. Bulbs of 11 Narcissus cultivars (Carlton, Cotinga, Exception, Geranium, Ice Follies, Ice King, Primeur, Tahiti, Tete-a-Tete, Thalia, and Westward) were planted into $15-\mathrm{cm}$ round plastic containers (three bulbs per container) filled with a soilless peat-based substrate (LM-111; Lambert Peat Moss, Inc., Riviere-Ouelle, Quebec, Canada), irrigated, and placed into a $9{ }^{\circ} \mathrm{C}$ cooler for 18 weeks. Over 6 weeks, temperature was gradually reduced to $1^{\circ} \mathrm{C}$ as rooting and shoot growth ensued. Containers were transferred to a glassglazed greenhouse on $8 \mathrm{Feb}$. and $1 \mathrm{Mar}$. and grown at $17{ }^{\circ} \mathrm{C}$ constant temperature. Plants 
Table 1. Effect of ethephon drenches on 11 Narcissus cultivars in 15-cm pots grown at Cornell. ${ }^{z}$

\begin{tabular}{|c|c|c|c|c|c|c|c|}
\hline $\begin{array}{l}\text { Ethephon concn } \\
\left(\mathrm{mg} \cdot \mathrm{L}^{-1}\right)\end{array}$ & $\begin{array}{l}\text { Time to first open } \\
\text { flower (days) }\end{array}$ & \multicolumn{2}{|c|}{$\begin{array}{l}\text { Length at first } \\
\text { open flower }(\mathrm{cm})\end{array}$} & \multicolumn{2}{|c|}{$\begin{array}{l}\text { Length at end of } \\
\text { flowering }(\mathrm{cm})\end{array}$} & \multicolumn{2}{|c|}{$\begin{array}{l}\text { Growth during } \\
\text { postharvest }(\mathrm{cm})\end{array}$} \\
\hline & & & & Iton' & & & \\
\hline 250 & 19 & 21.7 & 24.0 & 34.4 & 33.8 & 12.7 & 9.9 \\
\hline 500 & 18 & 18.9 & 22.8 & 28.3 & 29.9 & 9.4 & 7.1 \\
\hline \multirow[t]{2}{*}{ Significance } & $\mathrm{L}^{* * *} \mathrm{Q} * * *$ & $\mathrm{~L} * * * \mathrm{Q} * * *$ & $\mathrm{~L}^{* * *} \mathrm{Q}^{*}$ & $\mathrm{~L}^{* * *} \mathrm{Q} * * *$ & $\mathrm{~L}^{* * *} \mathrm{Q}^{*}$ & $\mathrm{~L} * * *$ & $\mathrm{~L}^{* * *}$ \\
\hline & \multicolumn{7}{|c|}{ 'Cotinga' } \\
\hline 100 & 15 & 23.0 & 26.7 & 31.8 & 31.4 & 8.8 & 4.7 \\
\hline 250 & 17 & 17.0 & 23.1 & 24.9 & 28.1 & 8.0 & 5.0 \\
\hline 500 & 16 & 15.1 & 21.8 & 22.2 & 26.5 & 7.1 & 4.8 \\
\hline \multirow[t]{2}{*}{ Significance } & $\mathrm{L}^{* *} \mathrm{Q}^{*}$ & $\mathrm{~L}^{* * *} \mathrm{Q}^{*}$ & $\mathrm{~L}^{* * *}$ & $\mathrm{~L}^{* * *} \mathrm{Q} * * *$ & $\mathrm{~L}^{* * *} \mathrm{Q}^{*}$ & $\mathrm{~L}^{* * *} \mathrm{Q}^{*}$ & NS \\
\hline & \multicolumn{7}{|c|}{ 'Exception' } \\
\hline 0 & 13 & 36.1 & 32.0 & 51.6 & 47.4 & 15.5 & 15.5 \\
\hline Significance & \multicolumn{7}{|c|}{ 'Geranium' } \\
\hline 0 & 19 & 32.6 & 32.4 & 62.5 & 48.5 & 30.0 & 16.2 \\
\hline 100 & 21 & 32.9 & 31.8 & 57.6 & 46.3 & 24.8 & 14.5 \\
\hline 250 & 27 & 29.5 & 32.4 & 49.1 & 38.38 & 19.7 & 6.5 \\
\hline 500 & 33 & 31.8 & 33.2 & 41.2 & 35.9 & 9.5 & 2.7 \\
\hline \multirow[t]{2}{*}{ Significance } & $\mathrm{L}^{* * *} \mathrm{QNS}$ & NS & NS & $\mathrm{L}^{* * *}$ & $\mathrm{~L}^{* * *}$ & $\mathrm{~L}^{* * *}$ & $\mathrm{~L}^{* * *}$ \\
\hline & \multicolumn{7}{|c|}{ 'Ice Follies' } \\
\hline 0 & 11 & 29.4 & 27.0 & 45.8 & 39.9 & 16.5 & 13.0 \\
\hline 100 & 12 & 25.7 & 24.4 & 36.9 & 34.9 & 11.2 & 10.6 \\
\hline 250 & 12 & 21.5 & 21.6 & 28.3 & 30.6 & 6.8 & 9.0 \\
\hline 500 & 13 & 19.9 & 20.3 & 24.5 & 26.9 & 4.6 & 6.6 \\
\hline Significance & LNSQNS & $\mathrm{L} * * * \mathrm{Q} * * *$ & $\mathrm{~L}^{* * *}$ & $\mathrm{~L}^{* * *} \mathrm{Q}^{* * *}$ & $\mathrm{~L}^{* * *}$ & $\mathrm{~L}^{* * *}$ & $\mathrm{~L}^{* * *}$ \\
\hline 100 & 18 & 25.6 & 25.0 & 35.6 & 35.6 & 10.1 & 10.6 \\
\hline 250 & 19 & 21.8 & 22.9 & 30.2 & 30.3 & 8.4 & 7.4 \\
\hline 500 & 20 & 19.6 & 20.3 & 25.1 & 26.4 & 6.1 & 6.3 \\
\hline Significance & $\mathrm{L}^{*} \mathrm{Q}_{\mathrm{NS}}$ & $\mathrm{L}^{* * *} \mathrm{Q} * * *$ & $\mathrm{~L}^{* * *}$ & $\mathrm{~L}^{* * *} \mathrm{Q}^{* * *}$ & $\mathrm{~L}^{* * *} \mathrm{Q}^{*}$ & $\mathrm{~L}^{* * *}$ & $\mathrm{~L}^{* * *}$ \\
\hline & & & & hiti' & & & \\
\hline 0 & 18 & 38.8 & 37.4 & 47.9 & 43.6 & 9.1 & 6.2 \\
\hline 100 & 19 & 33.3 & 33.9 & 41.2 & 40.5 & 8.0 & 6.6 \\
\hline 250 & 19 & 27.4 & 28.1 & 33.6 & 33.5 & 6.3 & 5.4 \\
\hline 500 & 21 & 25.2 & 25.5 & 28.6 & 28.2 & 3.4 & 2.8 \\
\hline Significance & $\mathrm{L}^{*} \mathrm{QNS}_{\mathrm{NS}}$ & $\mathrm{L} * * * \mathrm{Q} * * *$ & $\mathrm{~L}^{* * *} \mathrm{Q}^{* *}$ & $\mathrm{~L}^{* * *} \mathrm{Q} * * *$ & $\mathrm{~L}^{* * *}$ & $\mathrm{~L}^{* * *}$ & NS \\
\hline & & & & a-Tete' & & & \\
\hline 0 & 7 & 17.3 & 12.6 & 31.2 & 31.2 & 14.0 & 18.6 \\
\hline 100 & 7 & 16.1 & 13.1 & 26.7 & 28.3 & 10.6 & 12.4 \\
\hline 250 & 8 & 12.3 & 10.1 & 21.2 & 23.0 & 8.9 & 12.9 \\
\hline 500 & 8 & 11.5 & 11.0 & 16.3 & 19.2 & 4.8 & 8.3 \\
\hline Significance & LNSQNS & $\mathrm{L}^{* * *}$ & $\mathrm{~L}^{* *}$ & $\mathrm{~L}^{* * *} \mathrm{Q}^{* *}$ & $\mathrm{~L}^{* * *}$ & $\mathrm{~L}^{* * *}$ & $\mathrm{~L}^{* *}$ \\
\hline & & & & alia' & & & \\
\hline 0 & 25 & 34.4 & 30.0 & 44.7 & 34.4 & 10.4 & 4.4 \\
\hline 100 & 28 & 29.3 & 29.1 & 37.7 & 30.6 & 8.4 & 3.5 \\
\hline 250 & - $^{\mathrm{w}}$ & - & - & - & 27.3 & - & - \\
\hline 500 & - & - & - & - & 20.9 & - & - \\
\hline Significance & - & - & - & - & - & - & - \\
\hline
\end{tabular}


Table 1. (Continued) Effect of ethephon drenches on 11 Narcissus cultivars in 15-cm pots grown at Cornell. ${ }^{\mathrm{z}}$

\begin{tabular}{|c|c|c|c|c|c|c|c|}
\hline $\begin{array}{l}\text { Ethephon concn } \\
\left(\mathrm{mg} \cdot \mathrm{L}^{-1}\right)\end{array}$ & $\begin{array}{l}\text { Time to first open } \\
\text { flower (days) }\end{array}$ & \multicolumn{2}{|c|}{$\begin{array}{c}\text { Length at first } \\
\text { open flower }(\mathrm{cm})\end{array}$} & \multicolumn{2}{|c|}{$\begin{array}{l}\text { Length at end of } \\
\text { flowering }(\mathrm{cm})\end{array}$} & \multicolumn{2}{|c|}{$\begin{array}{l}\text { Growth during } \\
\text { postharvest }(\mathrm{cm})\end{array}$} \\
\hline & \multicolumn{7}{|c|}{ 'Westward' } \\
\hline 250 & 17 & 15.8 & 22.4 & 25.4 & 31.3 & 9.7 & 8.9 \\
\hline 500 & 18 & 14.3 & 22.4 & 19.4 & 28.6 & 5.1 & 6.3 \\
\hline Significance & $\mathrm{L}^{* *} \mathrm{Q}$ NS & $\mathrm{L}^{* * *} \mathrm{Q} * *$ & $\mathrm{~L} * *$ & $\mathrm{~L}^{* * *} \mathrm{Q} * *$ & $\mathrm{~L}^{* * *}$ & $\mathrm{~L}^{* * *}$ & $\mathrm{~L}^{*}$ \\
\hline
\end{tabular}

${ }^{2}$ All plants had 18 cold weeks of cold and drenched with $120 \mathrm{~mL}$ of solution containing $0,100,250$, or $500 \mathrm{mg} \cdot \mathrm{L}^{-1}$ ethephon when plants were 6 to $10 \mathrm{~cm}$ tall.

${ }^{y}$ From container rim to top of tallest bud or leaf, respectively.

${ }^{\mathrm{x}}$ (End of flowering) - (bud color).

wMany aborted flowers with these treatments.

Significance of linear (L) or quadratic (Q) regression: $\mathrm{NS}^{*},{ }^{*}, * * * *$ denotes nonsignificant or significant at $P \leq 0.05,0.01$, or 0.001 , respectively.

were irrigated as needed with municipal tap water (alkalinity $112 \mathrm{mg} \cdot \mathrm{L}^{-1} \mathrm{CaCO}_{3}$ ). When shoots were $6-10 \mathrm{~cm}$ long, $120-\mathrm{mL}$ drenches of $0,100,250$, or $500 \mathrm{mg} \cdot \mathrm{L}^{-1}$ ethephon (Florel; Lawn and Garden Products, Inc., Fresno, CA) were applied to each container providing $0,12,30$, and $60 \mathrm{mg}$ ethephon per container.

As the first flower per container opened, data were recorded and stem and leaf length (container rim to top of each) were recorded. When the crop was showing one senesced flower per container, final data on stem and leaf length were recorded. Growth (stem or leaf elongation) during this postharvest period was calculated by difference. There were five replicates for each cultivar. Data were analyzed using regression analyses using JMP (SAS, Cary, NC).

Expt. 2: Bedding plants at Cornell. On 22 Mar., seedlings of Catharanthus Roseus (L.) G. Don. 'Cooler Grape', Tagetes patula L. 'Crested Janie Deep Orange' [in 512-cell size (3.1-mL volume) plug trays], Celosia argentea L. var. plumosa Voss. 'New Look', Dianthus chinensis L. 'Super Parfait Raspberry', Petunia $\times$ hybrida Vilm. 'Single Dreams Midnight', Viola cornuta L. 'Penny Lane Mixed' [in 288-cell size (6-mL volume) plug trays], and Angelonia angustifolia Benth. 'Serena Lavendar', Pelargonium $\times$ hortorum L.H. Bailey 'Pinto Red' [in 128-cell size (12-mL volume) plug trays] and rooted cuttings of Antirrhinum majus L. 'Dragon Buttery' and Osteospermum fruticosium L. 'Zion Copper Amethyst' [in 51-cell size (27.2-mL volume) strips] were obtained from a commercial greenhouse (C. Raker \& Sons, Litchfield, $\mathrm{MI})$. Individual plants were transplanted into $10-\mathrm{cm}$ containers (495-mL volume) filled with a soilless peat-based substrate (LM-111; Lambert Peat Moss, Inc.). Plants were irrigated as needed with municipal tap water (alkalinity $112 \mathrm{mg} \cdot \mathrm{L}^{-1} \mathrm{CaCO}_{3}$ ) supplemented with water-soluble fertilizer to provide $150 \mathrm{mg} \cdot \mathrm{L}^{-1}$ nitrogen (N) (Jack's Professional LXTM Water Soluble Fertilizer All-Purpose 20N-2.2P-16.6K; J.R. Peter's Inc., Allentown, PA) with $30 \mathrm{mg} \cdot \mathrm{L}^{-1}$ magnesium added from $\mathrm{MgSO}_{4} \cdot 7 \mathrm{H}_{2} \mathrm{O}$. Two d after transplant, ethephon was applied as a $70-\mathrm{mL}$ substrate drench at concentrations of $0,25,50,100$, or $200 \mathrm{mg} \cdot \mathrm{L}^{-1}$ providing $0,1.8,3.5,7$, or $14 \mathrm{mg}$ ethephon per container. Plants were grown in
Table 2. Days to flower and plant characteristics of 11 bedding plant crops grown at Cornell treated $2 \mathrm{~d}$ after transplant with a $70-\mathrm{mL}$ ethephon drench per container at $0,25,50,100$, and $200 \mathrm{mg} \cdot \mathrm{L}^{-1}$.

\begin{tabular}{|c|c|c|c|c|c|}
\hline $\begin{array}{l}\text { Ethephon concn } \\
\left(\mathrm{mg} \cdot \mathrm{L}^{1}\right)\end{array}$ & Days to flower & $\begin{array}{c}\text { Stem } \\
\text { length }(\mathrm{cm})\end{array}$ & $\begin{array}{c}\text { Shoot } \\
\text { dry mass (g) }\end{array}$ & $\begin{array}{c}\text { Root } \\
\text { dry mass (g) }\end{array}$ & $\mathrm{R}: \mathrm{S}$ ratio \\
\hline & & & Angelonia & & \\
\hline 0 & 37.5 & 30.3 & 3.85 & 0.76 & 0.20 \\
\hline 25 & 41.3 & 25.5 & 1.81 & 0.46 & 0.25 \\
\hline 50 & 44.2 & 19.6 & 1.35 & 0.24 & 0.18 \\
\hline 100 & 58.5 & 19.1 & 1.26 & 0.18 & 0.15 \\
\hline 200 & 58.5 & 14.3 & 1.24 & 0.14 & 0.11 \\
\hline \multirow[t]{2}{*}{ Significance } & $\mathrm{L}^{* * *} \mathrm{Q} * * *$ & $\mathrm{~L} * * * \mathrm{Q}^{* * *}$ & $\mathrm{~L}^{* * *} \mathrm{Q} * * *$ & $\mathrm{~L}^{* * *} \mathrm{Q} * * *$ & $\mathrm{~L}^{* * *} \mathrm{Q} * * *$ \\
\hline & & & Antirrhinum $^{2}$ & & \\
\hline 0 & 28.3 & 21.7 & 5.92 & - & - \\
\hline 25 & 24.0 & 19.4 & 3.43 & - & - \\
\hline 50 & 25.0 & 17.3 & 4.31 & - & - \\
\hline 100 & 25.0 & 17.9 & 2.96 & - & - \\
\hline 200 & 25.0 & 16.0 & 1.95 & - & - \\
\hline \multirow[t]{2}{*}{ Significance } & LNSQNS & $\mathrm{L}^{*} \mathrm{Q}^{*}$ & $\mathrm{~L}^{* * *} \mathrm{Q} * * *$ & & \\
\hline & & & Catharanthus & & \\
\hline 0 & 62.0 & 12.3 & 3.67 & 0.67 & 0.18 \\
\hline 25 & 62.0 & 11.9 & 2.28 & 0.42 & 0.19 \\
\hline 50 & 66.8 & 10.0 & 1.66 & 0.35 & 0.21 \\
\hline 100 & 70.0 & 7.8 & 1.02 & 0.21 & 0.21 \\
\hline 200 & 70.0 & 5.0 & 0.69 & 0.10 & 0.14 \\
\hline \multirow[t]{2}{*}{ Significance } & $\mathrm{L}^{* * *} \mathrm{Q}^{* * *}$ & $\mathrm{~L}^{* * *} \mathrm{Q} * * *$ & $\mathrm{~L}^{* * *} \mathrm{Q}^{* * *}$ & $\mathrm{~L}^{* * *} \mathrm{Q}^{* * *}$ & LNSQ* \\
\hline & & & Celosia & & \\
\hline 0 & 47.0 & 26.0 & 3.63 & 0.79 & 0.22 \\
\hline 25 & 49.5 & 16.7 & 2.47 & 0.63 & 0.26 \\
\hline 50 & 55.0 & 12.1 & 1.73 & 0.41 & 0.24 \\
\hline 100 & 55.0 & 10.7 & 1.36 & 0.27 & 0.20 \\
\hline 200 & 55.0 & 7.8 & 0.86 & 0.17 & 0.20 \\
\hline \multirow[t]{2}{*}{ Significance } & $\mathrm{L}^{* * *} \mathrm{Q} * * *$ & $\mathrm{~L}^{* * *} \mathrm{Q} * * *$ & $\mathrm{~L}^{* * *} \mathrm{Q}^{* * *}$ & $\mathrm{~L}^{* * *} \mathrm{Q}^{* * *}$ & LNSQNS \\
\hline & & & Dianthus $^{\mathrm{z}}$ & & \\
\hline 0 & 55.0 & 15.8 & 3.74 & - & - \\
\hline 25 & 55.0 & 15.3 & 3.21 & - & - \\
\hline 50 & 53.7 & 12.3 & 2.53 & - & - \\
\hline 100 & 55.0 & 10.3 & 1.73 & - & - \\
\hline 200 & 55.0 & 10.2 & 1.42 & - & - \\
\hline \multirow[t]{2}{*}{ Significance } & LNSQNS & $\mathrm{L} * * * \mathrm{Q}^{* * *}$ & $\mathrm{~L}^{* * *} \mathrm{Q} * * *$ & & \\
\hline & & & Lobelia $^{\mathrm{zy}}$ & & \\
\hline 0 & - & 15.3 & 10.38 & - & - \\
\hline 25 & - & 13.8 & 10.67 & - & - \\
\hline 50 & - & 14.3 & 6.94 & - & - \\
\hline 100 & - & 13.3 & 6.53 & - & - \\
\hline 200 & - & 13.3 & 6.60 & - & - \\
\hline \multirow[t]{2}{*}{ Significance } & & LnsQns & $\mathrm{L}^{* * *} \mathrm{Q}^{* * *}$ & & \\
\hline & & & Osteospermum & & \\
\hline 0 & 39.3 & 21.0 & 4.81 & 1.06 & 0.18 \\
\hline 25 & 55.0 & 18.8 & 3.44 & 0.88 & 0.26 \\
\hline 50 & 52.3 & 18.5 & 3.52 & 0.93 & 0.23 \\
\hline 100 & 56.2 & 17.0 & 3.03 & 0.70 & 0.23 \\
\hline 200 & 55.0 & 14.3 & 2.21 & 0.51 & 0.23 \\
\hline Significance & $\mathrm{L}^{*} \mathrm{Q}^{* *}$ & $\mathrm{~L}^{* * *} \mathrm{Q} * * *$ & $\mathrm{~L}^{* * *} \mathrm{Q} * * *$ & $\mathrm{~L}^{* * *} \mathrm{Q} * * *$ & LNSQNS \\
\hline
\end{tabular}

(Continued on next page) 
Table 2. (Continued) Days to flower and plant characteristics of 11 bedding plant crops grown at Cornell treated $2 \mathrm{~d}$ after transplant with a $70-\mathrm{mL}$ ethephon drench per container at $0,25,50,100$, and 200 $\mathrm{mg} \cdot \mathrm{L}^{-1}$.

\begin{tabular}{|c|c|c|c|c|c|}
\hline $\begin{array}{l}\text { Ethephon concn } \\
\left(\mathrm{mg} \cdot \mathrm{L}^{1}\right)\end{array}$ & Days to flower & $\begin{array}{c}\text { Stem } \\
\text { length }(\mathrm{cm})\end{array}$ & $\begin{array}{c}\text { Shoot } \\
\text { dry mass }(g)\end{array}$ & $\begin{array}{c}\text { Root } \\
\text { dry mass (g) }\end{array}$ & $\mathrm{R}: \mathrm{S}$ ratio \\
\hline & & & Pelargonium $^{2}$ & & \\
\hline 0 & 64.7 & 28.8 & 12.85 & - & - \\
\hline 25 & 75.0 & 26.7 & 11.34 & - & - \\
\hline 50 & 75.0 & 24.7 & 10.33 & - & - \\
\hline 100 & 80.3 & 22.8 & 10.92 & - & - \\
\hline 200 & 83.0 & 19.8 & 8.52 & - & - \\
\hline \multirow[t]{2}{*}{ Significance } & $\mathrm{L}^{* * *} \mathrm{Q}^{* * *}$ & $\mathrm{~L}^{* * *} \mathrm{Q} * * *$ & $\mathrm{~L}^{* * *} \mathrm{Q}^{* * *}$ & & \\
\hline & & & Petunia ${ }^{2}$ & & \\
\hline 0 & 38.0 & 20.5 & 8.27 & - & - \\
\hline 25 & 46.2 & 21.1 & 4.02 & - & - \\
\hline 50 & 56.2 & 14.3 & 2.92 & - & - \\
\hline 100 & 63.3 & 14.5 & 2.87 & - & - \\
\hline 200 & 68.7 & 14.7 & 2.96 & - & - \\
\hline \multirow[t]{2}{*}{ Significance } & $\mathrm{L}^{* * *} \mathrm{Q} * * *$ & $\mathrm{~L}^{* *} \mathrm{Q} * *$ & $\mathrm{~L}^{* *} \mathrm{Q}^{* * *}$ & & \\
\hline & & & Tagetes & & \\
\hline 0 & 27.0 & 10.1 & 3.10 & 0.46 & 0.15 \\
\hline 25 & 28.8 & 10.0 & 2.47 & 0.45 & 0.18 \\
\hline 50 & 27.0 & 9.5 & 2.20 & 0.37 & 0.17 \\
\hline 100 & 25.8 & 8.9 & 1.84 & 0.32 & 0.18 \\
\hline 200 & 39.0 & 8.3 & 1.57 & 0.21 & 0.14 \\
\hline \multirow[t]{2}{*}{ Significance } & $\mathrm{L}^{* * *} \mathrm{Q} * * *$ & $\mathrm{~L}^{* *} \mathrm{Q}^{*}$ & $\mathrm{~L}^{* * *} \mathrm{Q}^{* * *}$ & $\mathrm{~L}^{* * *} \mathrm{Q} * * *$ & LNSQ* \\
\hline & & & Viola & & \\
\hline 0 & 23.0 & 11.5 & 2.83 & 0.40 & 0.14 \\
\hline 25 & 33.0 & 11.3 & 2.05 & 0.29 & 0.15 \\
\hline 50 & 51.0 & 10.7 & 1.37 & 0.28 & 0.21 \\
\hline 100 & 49.0 & 9.1 & 1.20 & 0.19 & 0.17 \\
\hline 200 & 52.3 & 7.0 & 1.03 & 0.09 & 0.09 \\
\hline Significance & $\mathrm{L}^{* * *} \mathrm{Q} * * *$ & $\mathrm{~L}^{* * *} \mathrm{Q} * * *$ & $\mathrm{~L}^{* * *} \mathrm{Q}^{* * *}$ & $\mathrm{~L}^{* * *} \mathrm{Q} * * *$ & LNSQ* \\
\hline
\end{tabular}

${ }^{\mathrm{z}}$ Root data were not collected for these species.

${ }^{y}$ Plants were already in flower at transplanting so days to flower was not applicable.

$\mathrm{R}: \mathrm{S}$ ratio $=$ root to shoot ratio; $\mathrm{NS},{ }^{*}, * *, * * *$ denotes nonsignificant or significant at $P<0.05,0.01$, or 0.001 , respectively.

a glass-glazed greenhouse at a constant air temperature set point of $18{ }^{\circ} \mathrm{C}$ and ambient light.

The date of first open flower was recorded for each plant and time from transplant to first open flower was calculated. Within a species when the last plants flowered, the following data were collected for all treatments: plant height, root (RDM) and shoot dry mass (SDM), and root to shoot ratio (R:S) was calculated. There were six replicates for each cultivar and treatment, and each treatment was randomly placed among two greenhouse benches. Data were analyzed using linear and quadratic regression in JMP (SAS).

Expt. 3: Bedding plants at Purdue. On 12 July, rooted cuttings of Angelonia 'Angel Mist White Cloud', Argyranthemum frutescens (L.) Sch. Bip. 'Madeira Cherry Red', Calibrachoa Cerv. hybrid 'Cabaret Pink Hot', Diascia barberae Hook. F. 'Wink Coral', Nemesia fruticans (Thunb.) Benth. 'Aromatica Royal', Sutera cordata Roth. 'Abunda Giant White', and Verbena Ruiz $\times$ hybrida 'Aztec Violet' were transplanted into $10-\mathrm{cm}$ containers $(480-\mathrm{mL}$ volume) filled with a soilless peat-based substrate (Fafard Custom 1P; Conrad Fafard, Inc., Agawam, MA). Plants were irrigated as needed with acidified water supplemented with watersoluble fertilizer to provide $200 \mathrm{mg} \cdot \mathrm{L}^{-1} \mathrm{~N}$ supplied from a combination of two fertilizers (Peters Excel@ Cal-Mag 21N-2.2P-16.5K and Peters Excel@ $15 \mathrm{~N}-2.2 \mathrm{P}-12.5 \mathrm{~K}$; The
Scotts Co., Marysville, OH). Irrigation water was supplemented with $93 \%$ sulfuric acid (Brenntag, Reading, PA) at $0.08 \mathrm{ppm}$ to reduce alkalinity to $100 \mathrm{ppm}$ and $\mathrm{pH}$ to a range of 5.8 to 6.2. Ten $\mathrm{d}$ after transplant, ethephon was applied as a $60-\mathrm{mL}$ drench $(0,25,50,100$, or $200 \mathrm{mg} \cdot \mathrm{L}^{-1}$ ethephon) providing $1.5,3,6$, or 12 mg ethephon per container. Plants were grown in a glass-glazed greenhouse at a constant air temperature set point of $20^{\circ} \mathrm{C}$.

On 24 Aug., stem length from the substrate to the tip of the longest shoot was recorded. Plants were then harvested and RDM and SDM were recorded for each plant and total dry mass (TDM) and R:S ratio were calculated. There were six replicates for each cultivar and treatment. Regression analyses on data were performed using SPSS (Version 18.0; SPSS, Inc., Chicago, IL).

Expt. 4: Bedding plants at Michigan State University. On 12 May, seedlings of Lycopersicon esculentum L. 'Beefmaster', Petunia $\times$ grandiflora 'Dreams Burgundy' [in 512-cell size (3.1-mL volume) plug trays], Antirrhinum majus 'Montego Purple', and Impatiens walleriana Hook. f. 'Super Elfin Bright Orange' [in 288-cell size (6-mL volume) plug trays] and rooted cuttings of Osteospermum 'Zion Orange' [in 51-cell size (27.2-mL volume) strips] were obtained from a commercial greenhouse (C. Raker \& Sons, Litchfield, MI). Each was transplanted into 10 -cm containers ( $480-\mathrm{mL}$ volume) filled with a soilless peat-based substrate (Suremix;
Michigan Grower Products, Inc., Galesburg, MI). Plants were irrigated as needed with reverse osmosis water supplemented with a $14 \mathrm{~N}-3 \mathrm{P}-14 \mathrm{~K}$ water-soluble fertilizer (MSU RO Water Special; GreenCare Fertilizers, Inc., Kankakee, IL) to provide $125 \mathrm{mg} \cdot \mathrm{L}^{-1} \mathrm{~N}$. Ten $\mathrm{d}$ after transplant, ethephon concentrations of $0,50,100$, or $200 \mathrm{mg} \cdot \mathrm{L}^{-1}$ were prepared and applied as a $60-\mathrm{mL}$ drench providing 3,6 , or $12 \mathrm{mg}$ per container. Plants were grown in a glass-glazed greenhouse at a constant air temperature set point of $20^{\circ} \mathrm{C}$.

The date of first open flower was recorded for each plant and time from transplant to first open flower was calculated. When each plant flowered, the following data were collected: plant height, number of visible flowers and flower buds, lateral branch number on the primary stem, and visual root rating $(5=$ large, well developed; 1 = small, poorly developed). Plants that did not flower within $64 \mathrm{~d}$ after transplant were not included in the analysis. There were 10 replicates for each species and treatment. Data were analyzed with SAS (SAS Institute, Inc., Cary, NC) linear and quadratic regression procedure (PROC REG).

Expt. 5: Ethylene release from substrates of varying $\mathrm{pH}$ at Cornell. Substrates of different $\mathrm{pH}$ were prepared by adding 0 to $11.9 \mathrm{~kg}$ dolomitic lime to $1 \mathrm{~m}^{-3}$ of Canadian sphagnum peatmoss. The mixtures were slightly moistened with water, sealed to avoid moisture loss, and held at room temperature for 3 weeks. A volume of substrate equal to a 10 -cm container $(\approx 0.5 \mathrm{~L})$ was placed into 0.88 -L glass jars. Ethephon (100 $\mathrm{mg} \cdot \mathrm{L}^{-1}, 60-\mathrm{mL}$ volume) was added to the jars that were then sealed with lids fitted with rubber septa. Two controls consisting of ethephon liquid only $\left(100 \mathrm{mg} \cdot \mathrm{L}^{-1}, 60 \mathrm{~mL}\right)$ with or without 2 pellets of $\mathrm{KOH}$ were also used. Headspace samples of $1 \mathrm{~mL}$ were withdrawn at intervals and ethylene concentration determined by injection into a gas chromatograph equipped for ethylene analysis. There were five replicates per treatment.

\section{Results}

Expt. 1: Ethephon drenches on Narcissus at Cornell. There were few differences between the two experiments, so data were pooled for the final analysis. Ethephon drenches reduced stem and leaf growth across most Narcissus cultivars trialed (Table 1); this was observed at the time of first open flower and as plants began to senesce. With the ethephon concentrations used, there were significant reductions in additional stem (2- to 3-fold) and leaf (usually 2-fold) growth during the post-flowering period. Among all cultivars, ethephon drenches generally caused a 2- to 3-d flowering delay, especially at higher concentrations. There were no differences in duration of flowering or any evidence of negative effects of ethephon on flower longevity among most cultivars (data not presented). There was no visible foliar phytotoxicity at the concentrations and volume applied. However, two cultivars showed 
negative effects. Flowering of 'Geranium' was severely delayed by ethephon drenches, although it eventually flowered normally. Flower stem growth was restricted and flower abortion of 'Thalia' was observed with every ethephon treatment and exacerbated at the two highest concentrations.

Expt. 2: Bedding plants at Cornell. Ethephon drenches had no effect on time to flower for Antirrhinum, Dianthus, and Lobelia but delayed flowering of Angelonia, Catharanthus, Celosia, Osteospermum, Pelargonium, Petunia, Tagetes, and Viola (Table 2). Most notably, flowering was delayed by 30 and $31 \mathrm{~d}$ for Viola and Petunia, respectively, at $200 \mathrm{mg} \cdot \mathrm{L}^{-1}$ as compared with $0 \mathrm{mg} \cdot \mathrm{L}^{-1}$ ethephon drenches. Celosia, Osteospermum, and Viola flowered later as ethephon drench concentration increased from 0 to $50 \mathrm{mg} \cdot \mathrm{L}^{-1}$, whereas greater concentrations did not further delay flowering. Stem length was reduced by ethephon drenches for all species except Lobelia. Among the species most affected were Celosia, Catharanthus, and Angelonia with stem length reduction of $70 \%$, $59 \%$, and $53 \%$, respectively, at $200 \mathrm{mg} \cdot \mathrm{L}^{-1}$ vs. control plants. The SDM of all 11 species was negatively affected by increasing ethephon drench concentrations. Stem length was reduced in Catharanthus (81\%) and Celosia $(76 \%)$ in response to $200 \mathrm{mg} \cdot \mathrm{L}^{-1}$ ethephon, whereas the least affected species were $\mathrm{Pel}$ argonium (31\%) and Lobelia (36\%). Depending on species, reduction in RDM varied from $52 \%$ to $85 \%$ for Osteospermum and Catharanthus, respectively, for plants treated with drenches containing $200 \mathrm{mg} \cdot \mathrm{L}^{-1}$ ethephon. The R:S ratio of Celosia and Osteospermum was unaffected by increasing ethephon concentration, whereas Tagetes, Viola, and Catharanthus exhibited a quadratic response.

Expt. 3: Bedding plants at Purdue. As ethephon drench concentration increased from 0 to $200 \mathrm{mg} \cdot \mathrm{L}^{-1}$, final stem length decreased for nearly all species (Table 3 ). For example, when $200 \mathrm{mg} \cdot \mathrm{L}^{-1}$ ethephon was applied to the substrate of Angelonia, Calibrachoa, and Nemesia, stem length was $8.9,8.3$, and $11.6 \mathrm{~cm}$ shorter, respectively, compared with untreated plants. An ethephon drench had no effect on stem length of Diascia. Ethephon reduced TDM and SDM for all species as concentration increased and was greatest in trailing species such as Calibrachoa, Nemesia, Sutera, and Verbena. The TDM of Calibrachoa, Nemesia, Sutera, and Verbena treated with a $200 \mathrm{mg} \cdot \mathrm{L}^{-1}$ ethephon drench was reduced by $60 \%, 39 \%, 40 \%$, and $37 \%$, respectively, compared with control plants. Similarly, RDM of Calibrachoa, Diascia, Nemesia, and Sutera treated with $200 \mathrm{mg} \cdot \mathrm{L}^{-1}$ ethephon was reduced by $53 \%, 73 \%, 59 \%$, and $43 \%$, respectively; RDM of Angelonia and Verbena was unaffected. The R:S ratios of Calibrachoa, Nemesia, Osteospermum, and Sutera were not or marginally influenced by ethephon. In contrast, the $\mathrm{R}: \mathrm{S}$ ratio increased by $44 \%$ in Angelonia and decreased by $60 \%$ and $15 \%$ in Argyranthemum and Diascia, respectively, as the ethephon drench concentration increased to $200 \mathrm{mg} \cdot \mathrm{L}^{-1}$.

Table 3. Plant characteristics of eight bedding plant crops grown at Purdue treated $10 \mathrm{~d}$ after transplant with a $74-\mathrm{mL}$ ethephon drench per container at $25,50,100$, and $200 \mathrm{mg} \cdot \mathrm{L}^{-1}$.

\begin{tabular}{|c|c|c|c|c|c|}
\hline $\begin{array}{l}\text { Ethephon concn } \\
\left(\mathrm{mg} \cdot \mathrm{L}^{-1}\right)\end{array}$ & $\begin{array}{c}\text { Stem } \\
\text { length }(\mathrm{cm})\end{array}$ & $\begin{array}{c}\text { Total } \\
\text { dry mass (g) }\end{array}$ & $\begin{array}{c}\text { Shoot } \\
\text { dry mass (g) }\end{array}$ & $\begin{array}{c}\text { Root } \\
\text { dry mass (g) }\end{array}$ & $\mathrm{R}: \mathrm{S}$ ratio \\
\hline & & & Angelonia & & \\
\hline 0 & 40.4 & 7.35 & 6.35 & 1.00 & 0.16 \\
\hline 25 & 39.7 & 7.08 & 6.02 & 1.06 & 0.18 \\
\hline 50 & 36.6 & 6.83 & 6.00 & 0.83 & 0.14 \\
\hline 100 & 34.9 & 5.71 & 4.95 & 0.76 & 0.15 \\
\hline 200 & 31.5 & 5.13 & 4.23 & 0.90 & 0.23 \\
\hline \multirow[t]{2}{*}{ Significance } & $\mathrm{L}^{* * *} \mathrm{Q} * * *$ & $\mathrm{~L}^{* * *} \mathrm{Q}^{* *}$ & $\mathrm{~L}^{* * *} \mathrm{Q}^{* *}$ & LnsQns & $\mathrm{L}^{* *} \mathrm{Q}^{* *}$ \\
\hline & & & Argyranthemum & & \\
\hline 0 & 17.1 & 7.53 & 6.31 & 1.22 & 0.19 \\
\hline 25 & 16.1 & 6.88 & 5.90 & 0.98 & 0.17 \\
\hline 50 & 15.3 & 6.93 & 6.02 & 0.91 & 0.15 \\
\hline 100 & 14.1 & 5.06 & 4.42 & 0.64 & 0.14 \\
\hline 200 & 13.9 & 5.39 & 4.66 & 0.73 & 0.16 \\
\hline \multirow[t]{2}{*}{ Significance } & $\mathrm{L}^{* * *} \mathrm{Q} * * *$ & $\mathrm{~L}^{* * *} \mathrm{Q}^{* * *}$ & $\mathrm{~L} * * * \mathrm{Q} * * *$ & $\mathrm{~L}^{* * *} \mathrm{Q} * * *$ & $\mathrm{~L} * \mathrm{Q} * * *$ \\
\hline & & & Calibrachoa & & \\
\hline 0 & 25.9 & 5.68 & 5.32 & 0.36 & 0.07 \\
\hline 25 & 25.0 & 5.09 & 4.81 & 0.28 & 0.06 \\
\hline 50 & 23.6 & 3.47 & 3.22 & 0.25 & 0.08 \\
\hline 100 & 21.1 & 2.98 & 2.75 & 0.23 & 0.08 \\
\hline 200 & 17.6 & 2.26 & 2.09 & 0.17 & 0.09 \\
\hline \multirow[t]{2}{*}{ Significance } & $\mathrm{L}^{* * *} \mathrm{Q} * * *$ & $\mathrm{~L}^{* * *} \mathrm{Q}^{* * *}$ & $\mathrm{~L}^{* * *} \mathrm{Q} * * *$ & $\mathrm{~L}^{* * *} \mathrm{Q} * * *$ & $\mathrm{~L}^{*} \mathrm{Q}^{*}$ \\
\hline & & & Diascia & & \\
\hline 0 & 29.5 & 9.16 & 8.00 & 1.16 & 0.15 \\
\hline 25 & 35.4 & 8.91 & 8.20 & 0.71 & 0.09 \\
\hline 50 & 36.0 & 8.19 & 7.69 & 0.50 & 0.07 \\
\hline 100 & 34.5 & 7.29 & 6.91 & 0.38 & 0.06 \\
\hline 200 & 32.3 & 6.32 & 6.01 & 0.31 & 0.06 \\
\hline \multirow[t]{2}{*}{ Significance } & LNSQNS & $\mathrm{L}^{* * *} \mathrm{Q} * *$ & $\mathrm{~L}^{* *} \mathrm{Q} * *$ & $\mathrm{~L}^{* * *} \mathrm{Q} * * *$ & $\mathrm{~L}^{* *} \mathrm{Q} * * *$ \\
\hline & & & Nemesia & & \\
\hline 0 & 36.1 & 5.37 & 5.20 & 0.17 & 0.03 \\
\hline 25 & 37.5 & 4.52 & 4.38 & 0.14 & 0.03 \\
\hline 50 & 30.2 & 3.49 & 3.37 & 0.12 & 0.04 \\
\hline 100 & 26.9 & 3.31 & 3.21 & 0.10 & 0.03 \\
\hline 200 & 24.5 & 3.30 & 3.23 & 0.07 & 0.03 \\
\hline \multirow[t]{2}{*}{ Significance } & $\mathrm{L}^{* * *} \mathrm{Q} * * *$ & $\mathrm{~L}^{*} \mathrm{Q}^{* *}$ & $\mathrm{~L}^{*} \mathrm{Q}^{* *}$ & $\mathrm{~L}^{* * *} \mathrm{Q} * * *$ & LNSQNS \\
\hline & & & Osteospermum & & \\
\hline 0 & 22.7 & 7.16 & 6.39 & 0.78 & 0.12 \\
\hline 25 & 22.1 & 6.59 & 5.85 & 0.75 & 0.13 \\
\hline 50 & 19.5 & 5.21 & 4.70 & 0.52 & 0.11 \\
\hline 100 & 20.6 & 4.87 & 4.41 & 0.45 & 0.10 \\
\hline 200 & 19.1 & 5.12 & 4.60 & 0.52 & 0.12 \\
\hline \multirow[t]{2}{*}{ Significance } & $\mathrm{L}^{*} \mathrm{Q}^{*}$ & $\mathrm{~L}^{* *} \mathrm{Q}^{* * *}$ & $\mathrm{~L}^{* *} \mathrm{Q}^{* * *}$ & $\mathrm{~L}^{*} \mathrm{Q}^{* *}$ & LNSQNS \\
\hline & & & Sutera & & \\
\hline 0 & 40.0 & 10.72 & 10.20 & 0.51 & 0.05 \\
\hline 25 & 43.1 & 9.38 & 8.90 & 0.48 & 0.05 \\
\hline 50 & 37.8 & 9.54 & 9.03 & 0.51 & 0.06 \\
\hline 100 & 33.7 & 6.56 & 6.25 & 0.31 & 0.05 \\
\hline 200 & 34.3 & 6.41 & 6.12 & 0.29 & 0.05 \\
\hline \multirow[t]{2}{*}{ Significance } & $\mathrm{L}^{* * *} \mathrm{Q} * * *$ & $\mathrm{~L}^{* * *} \mathrm{Q} * * *$ & $\mathrm{~L}^{* * *} \mathrm{Q} * * *$ & $\mathrm{~L}^{* * *} \mathrm{Q} * * *$ & LNSQNS \\
\hline & & & Verbena & & \\
\hline 0 & 28.2 & 7.00 & 6.74 & 0.26 & 0.04 \\
\hline 25 & 29.8 & 6.47 & 6.15 & 0.32 & 0.05 \\
\hline 50 & 23.1 & 5.31 & 5.07 & 0.24 & 0.05 \\
\hline 100 & 19.3 & 4.04 & 3.79 & 0.25 & 0.06 \\
\hline 200 & 24.9 & 4.38 & 4.17 & 0.21 & 0.05 \\
\hline Significance & LnsQ* & $\mathrm{L}^{* *} \mathrm{Q} * *$ & $\mathrm{~L}^{* *} \mathrm{Q} * *$ & LNSQNS & LnsQ* \\
\hline
\end{tabular}

$\mathrm{R}: \mathrm{S}$ ratio $=$ root to shoot ratio; Significance of linear $(\mathrm{L})$ or quadratic $(\mathrm{Q})$ regression: $\mathrm{NS},{ }^{*},{ }^{* *},{ }^{* * *}$ denotes nonsignificant or significant at $P \leq 0.05,0.01$, or 0.001 , respectively.

Expt. 4: Bedding plants at Michigan State University. Ethephon drenches had no effect on flowering time of Antirrhinum, Impatiens, or Osteospermum but increasingly delayed flowering in Lycopersicon and Petunia as concentration increased from 0 to $200 \mathrm{mg} \cdot \mathrm{L}^{-1}$ (Table 4). In Lycopersicon, the 100 and especially $200-\mathrm{mg} \cdot \mathrm{L}^{-1}$ drench treatments caused stem epinasty and promoted adventi- tious rooting along stems (data not collected). Plant height at flowering decreased as the ethephon concentration increased in Antirrhinum (up to $12 \%$ ) and Impatiens (up to $22 \%$ ), but there was no consistent effect on the other three crops. Ethephon had contrasting effects on flower or inflorescence number at first flowering, showing a quadratic response with Antirrhinum, a decreasing response (up to $60 \%$ 
Table 4. Days to flower and plant characteristics at flowering of five bedding plant crops grown at Michigan State University treated $10 \mathrm{~d}$ after transplant with a 60-mL ethephon drench per container at $0,50,100$, and $200 \mathrm{mg} \cdot \mathrm{L}^{-1}$.

\begin{tabular}{|c|c|c|c|c|c|}
\hline $\begin{array}{l}\text { Ethephon concn } \\
\left(\mathrm{mg} \cdot \mathrm{L}^{-1}\right)\end{array}$ & Days to flower & Ht at flower $(\mathrm{cm})$ & $\begin{array}{l}\text { Number of } \\
\text { branches }\end{array}$ & $\begin{array}{l}\text { Number of } \\
\text { flowers of } \\
\text { inflorescences }\end{array}$ & Root rating \\
\hline & \multicolumn{5}{|c|}{ Antirrhinum } \\
\hline 0 & 28.2 & 13.3 & 13.8 & 43.7 & 1.70 \\
\hline 50 & 28.4 & 13.1 & 14.3 & 54.3 & 1.90 \\
\hline 100 & 29.6 & 12.3 & 14.0 & 39.3 & 2.30 \\
\hline 200 & 29.7 & 11.7 & 13.8 & 33.5 & 1.80 \\
\hline \multirow[t]{2}{*}{ Significance } & LNSQNS & $\mathrm{L}^{* *} \mathrm{Q} *$ & LNSQNS & $\mathrm{L}^{*} \mathrm{Q}^{*}$ & LNsQ* \\
\hline & \multicolumn{5}{|c|}{ Impatiens } \\
\hline 0 & 33.5 & 6.57 & 4.20 & 60.0 & 3.40 \\
\hline 50 & 31.1 & 5.24 & 4.10 & 42.4 & 2.60 \\
\hline 100 & 33.2 & 5.34 & 4.20 & 33.8 & 2.30 \\
\hline 200 & 34.0 & 5.14 & 4.00 & 24.2 & 1.90 \\
\hline \multirow[t]{2}{*}{ Significance } & LNSQNS & $\mathrm{L}^{* *} \mathrm{Q} * *$ & LNSQNS & $\mathrm{L} * * * \mathrm{Q} * * *$ & $\mathrm{~L} * * * \mathrm{Q} * * *$ \\
\hline & \multicolumn{5}{|c|}{ Lycopersicon } \\
\hline 0 & 37.4 & 64.1 & 11.3 & 9.20 & 4.60 \\
\hline 50 & 38.1 & 52.8 & 10.6 & 12.2 & 4.60 \\
\hline 100 & 44.4 & 56.6 & 12.6 & 15.1 & 4.90 \\
\hline 200 & 55.8 & 58.8 & 11.3 & 17.4 & 2.60 \\
\hline \multirow[t]{2}{*}{ Significance } & $\mathrm{L}^{* * *} \mathrm{Q}^{* * *}$ & $\mathrm{LNSQ}^{* *}$ & LNSQNS & $\mathrm{L}^{* * *} \mathrm{Q} * * *$ & $\mathrm{~L} * * * \mathrm{Q}^{* * *}$ \\
\hline & \multicolumn{5}{|c|}{ Osteospermum } \\
\hline 0 & 51.1 & 22.2 & 37.1 & 13.7 & 1.90 \\
\hline 50 & 51.6 & 23.2 & 35.7 & 16.4 & 2.70 \\
\hline 100 & 54.2 & 21.4 & 36.6 & 19.6 & 1.40 \\
\hline 200 & 52.8 & 21.0 & 35.7 & 19.9 & 1.70 \\
\hline \multirow[t]{2}{*}{ Significance } & LNSQNS & LNSQNS & LNSQNS & $\mathrm{L} * * * \mathrm{Q} * * *$ & LNSQNS \\
\hline & \multicolumn{5}{|c|}{ Petunia } \\
\hline 0 & 28.1 & 8.69 & 10.7 & 7.10 & 1.70 \\
\hline 50 & 29.1 & 7.80 & 11.7 & 8.90 & 2.70 \\
\hline 100 & 36.4 & 8.94 & 11.9 & 16.5 & 2.40 \\
\hline 200 & 42.3 & 9.67 & 11.0 & 20.3 & 2.10 \\
\hline Significance & $\mathrm{L} * * * \mathrm{Q} * * *$ & LNSQNS & LNSQNS & $\mathrm{L}^{* * *} \mathrm{Q} * * *$ & LNSQ* \\
\hline
\end{tabular}

${ }^{z}$ The subjective root rating was performed at flowering and ranged from 5 (large, well developed) to 1 (small, poorly developed). Significance of linear (L) or quadratic (Q) regression: Ns, *, **, *** denotes nonsignificant or significant at $P \leq 0.05,0.01$, or 0.001 , respectively. fewer flowers) in Impatiens, and an increasing response in Lycopersicon, Osteospermum, and Petunia (up to $90 \%, 45 \%$, and $186 \%$ more flowers, respectively). The root quality ratings at first flowering were variable and there were no consistent trends among the five crops studied.

Expt. 5: Ethylene release from substrates of varying $\mathrm{pH}$. Within $\approx 120 \mathrm{~h}$, maximal ethylene release in the container was measured with ethephon and $\mathrm{KOH}$ and final ethylene concentrations were close to the theoretical concentration of $\approx 1045 \mu \mathrm{L} \cdot \mathrm{L}^{-1}$ (Fig. 1). Essentially no ethylene (less than $1 \mu \mathrm{L} \cdot \mathrm{L}^{-1}$ ) was released from ethephon in the absence of $\mathrm{KOH}$ or substrate. Ethylene release from peatmoss was dramatically increased by incorporating dolomitic lime (Table 5). When no lime was added to the substrate, very little ethylene accumulated (less than $20 \mu \mathrm{L} \cdot \mathrm{L}^{-1}$ ), although this concentration was significantly greater than the container with only ethephon. As lime addition increased to $9.5 \mathrm{~kg} \cdot \mathrm{L}^{-1}$, ethylene release and accumulation in the headspace increased.

\section{Discussion}

Ethephon drenches inhibited stem growth in nearly all of the bedding plants studied, although the magnitude varied among species and locations. For example, a 60- to 70-mL drench per $10-\mathrm{cm}$ container of $100 \mathrm{mg} \cdot \mathrm{L}^{-1}$ ethephon solution reduced plant height at flowering by greater than 30\% in Angelonia (at Cornell), Catharanthus, Celosia, Dianthus, and Verbena but by only $10 \%$ to $15 \%$ in Angelonia (at Purdue), Lobelia, Lycopersicon, and Tagetes (Fig. 2). Generally, the height suppression was greatest in the study at Cornell, which could be attributed to the earlier

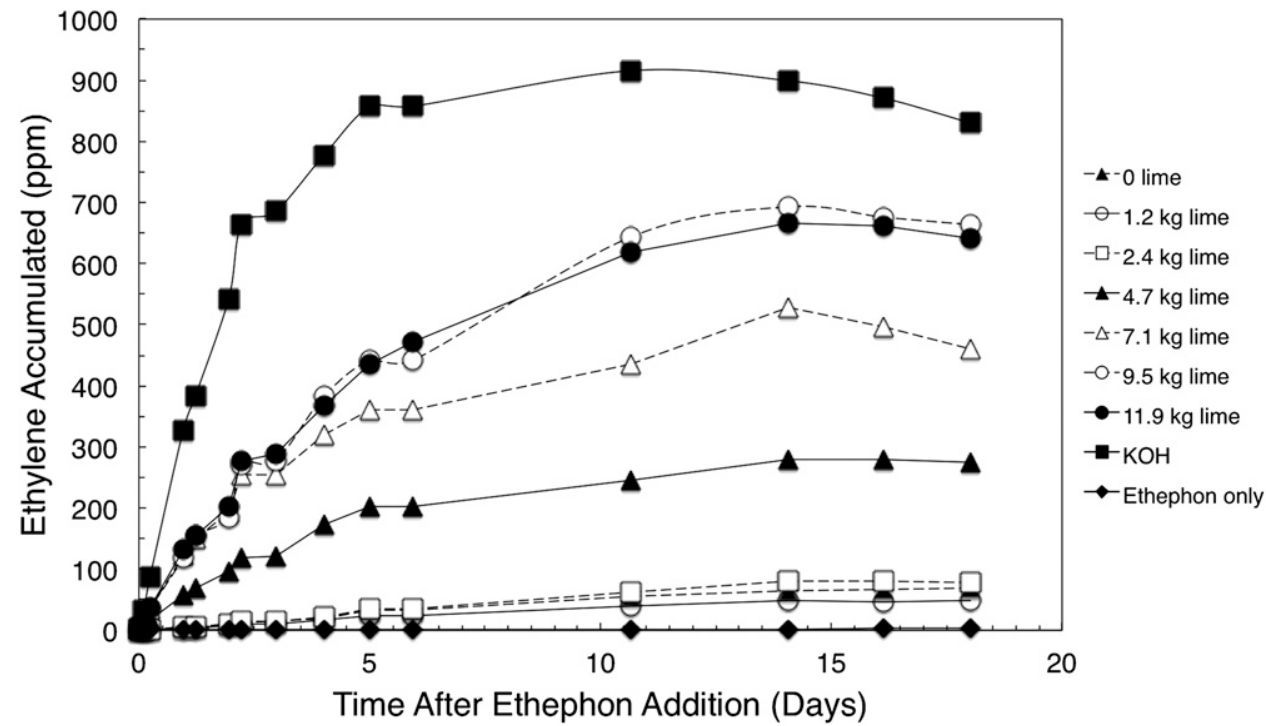

Fig. 1. Effect of lime addition to peatmoss on ethylene evolution after ethephon application. Substrates were prepared by mixing 0 to $11.9 \mathrm{~kg}$ dolomitic lime to $1 \mathrm{~m}^{3}$ of sphagnum peatmoss, moistened slightly, sealed, then held at room temperature in darkness for 3 weeks. A volume of substrate equivalent to that held in a $10-\mathrm{cm}$ pot was placed in a 0.88 - L glass jar, moistened with tap water, then $60 \mathrm{~mL}$ of solution containing $100 \mathrm{mg} \cdot \mathrm{L}^{-1}$ ethephon was added to each jar. Jars were sealed and 1-mL samples withdrawn at varying intervals for ethylene analysis by gas chromatography. Additional controls (without peat) consisted of $60 \mathrm{~mL}$ of $100 \mathrm{mg} \cdot \mathrm{L}^{-1}$ ethephon with or without 2 pellets of $\mathrm{KOH}$ added. Points are means of three to five replicates. 
application (2 d after transplant) compared with the other locations ( $10 \mathrm{~d}$ after transplant). For most crops, stem length decreased linearly or quadratically with increasing ethephon application. Exceptions were Lobelia at Cornell, Diascia at Purdue, and Osteospermum and Petunia at Michigan State University, which showed no significant trends. It is likely the response to a given ethephon drench will depend on many variables, perhaps including species and cultivar, drench timing, temperature, light, substrate components, and other factors. To our knowledge, this is the first published report on the effects of an ethephon drench on bedding plants and thus, comparisons with other studies are not possible.

Flowering time of bedding plants was quantified at Cornell and Michigan State University and an ethephon drench delayed flowering in 10 of the 16 crops, especially

Table 5. pH of sphagnum peat amended with dolomitic lime. ${ }^{z}$

\begin{tabular}{lc}
\hline Lime $\left(\mathrm{kg} \cdot \mathrm{m}^{-3}\right)$ & $\mathrm{pH}$ \\
\hline 0 & 3.86 \\
1.2 & 4.58 \\
2.4 & 5.10 \\
4.7 & 6.52 \\
7.1 & 6.87 \\
9.5 & 7.13 \\
11.9 & 7.27 \\
\hline
\end{tabular}

${ }^{\mathrm{z}}$ Means of five replications.



Fig. 2. The effect of a drench containing $100 \mathrm{mg} \cdot \mathrm{L}^{-1}$ ethephon on percentage height reduction in bedding plants grown in $10-\mathrm{cm}$ containers at three universities. The drench volume per pot and time of application (days after transplant) were $70 \mathrm{~mL}$ and $2 \mathrm{~d}$ at Cornell and $60 \mathrm{~mL}$ and $10 \mathrm{~d}$ at Purdue and Michigan State University (MSU). Ethephon had no effect on plant height of Diascia at Purdue or Petunia at MSU.

at concentrations $100 \mathrm{mg} \cdot \mathrm{L}^{-1}$ or greater. For example, a $200-\mathrm{mg} \cdot \mathrm{L}^{-1}$ ethephon drench delayed flowering of Petunia by $81 \%$ at Cornell and by $51 \%$ at Michigan State University. Ethephon drenches had no effect on flowering time of Antirrhinum at either location and Dianthus, Impatiens, or Osteospermum only at Michigan State University. Ethephon drenches inhibited SDM in all crops in which it was quantified at Cornell and Purdue. The RDM was also suppressed in most of these crops, resulting in somewhat inconsistent $\mathrm{R}: \mathrm{S}$ ratios. Ethephon had a contrasting effect on flower number at Michigan State University. Increasing ethephon concentration dramatically decreased flower numbers in Impatiens but increased flowers in Lycopersicon, Osteospermum, and Petunia.

The present experiments show potential for the use of ethephon as a substrate drench on Narcissus. Unlike the bedding plant studies, we did not evaluate root growth of Narcissus, because nearly all root growth occurred during cooling before ethephon was applied. Thus, in Narcissus, the growth reductions are not the result of restricted root growth and are more likely a direct result of the ethylene released from ethephon. In the Narcissus forcing industry, ethephon sprays are the most common method of height control, but they are not always effective (Moe, 1980; Miller, personal observation) and this

\section{(n)}

has prompted more recent research into using bulb dips and substrate drenches containing flurprimidol, paclobutrazol, and uniconazole for height control (Krug, et al., 2005, 2006a, 2006b; Miller, 2011). The present results demonstrate that excellent height control of Narcissus using ethephon substrate drenches may be achieved. This suggests that variability in Narcissus response to ethephon sprays may be the result of spray application technique such as the volume of solution applied per container or area. Perhaps much of the height-suppressing effect of ethephon sprays on Narcissus is actually the result of a substrate drench effect and greater efficacy is seen when greater spray volumes are used.

Although our results demonstrate that ethephon applied to substrate can be an effective growth retardant, the exact mechanism by which this occurs is unknown. Ethephon drenches reduced RDM, root ratings, or R:S ratios in several bedding plants in this study (Tables 2 to 4). However, similar ethephon concentrations reduced plant height when applied to both fully rooted Narcissus plants (Table 1) or newly transplanted bedding plants (Tables 2 to 4). Therefore, reduced root growth or development cannot be the primary mechanism by which inhibited stem growth occurred in Narcissus. One mechanism may be that ethylene released in substrate diffuses toward the shoot over a prolonged period of time, where it suppresses cell elongation. This hypothesis is supported by data from Expt. 5 showing long-term release of ethylene from substrates of varying $\mathrm{pH}$ (Fig. 1) and by the fact Narcissus leaves elongate as a result of cell divisions in the intercalary meristem with further elongation occurring within $\approx 40 \mathrm{~mm}$ of the leaf base (Denne, 1960). In most forcing situations, these zones would be under the substrate surface and exposed to substrate borne ethylene or direct contact with ethephon. Alternatively, some of the applied ethephon may be translocated in the plant, as has been demonstrated (Edgerton and Hatch, 1972; Martin et al., 1972). Such translocated ethephon could then release ethylene in subapical elongation regions, leading to inhibited stem growth. It is unknown, however, if ethephon can be absorbed into intact roots of the plants investigated or the overall extent of translocation (if any). Clearly, additional work is needed to elucidate the mode(s) of action of substrate-applied ethephon. The effect of liming on ethylene release suggests a possible experimental approach whereby rate of ethylene generation may be modified by substrate $\mathrm{pH}$.

Despite the potential benefits of ethephon drenches in terms of regulation of stem extension, our findings demonstrate several unwanted side effects for many of the bedding plant crops. The reduction in root and shoot development was most dramatic when drenches were applied $2 \mathrm{~d}$ after transplant as opposed to $10 \mathrm{~d}$ after transplant. It was also noted that morphology of Lycopersicon plants was significantly affected by ethephon drenches. Treated plants exhibited severely bent stems and adventitious rooting on stems (Runkle, 
personal observation), similar to the characteristic "triple response" to ethylene (Reid, 1987). A delay in flowering due to ethephon drenches was found in many bedding plant crops, although species varied in their sensitivity (Tables 2 and 4) and this finding was not unexpected. Although some flowers are insensitive to ethylene, flowering may be promoted by ethylene such as in bromeliads (Dolan, 1997). However, exogenous applications of ethylene are well known to affect various aspects of flower development including flower bud abortion, delay in floral initiation, senescence of mature flower buds, wilting of petals, or failure of the bud to open (Reid, 1987).

One of the drawbacks of ethephon drenches in bedding plant production is a delay in flowering. However, consideration must also be given to the potential effect of ethephon drenches on postharvest flower life and senescence when drenches are made late in crop production. Ethephon drenches of 100 or $200 \mathrm{mg} \cdot \mathrm{L}^{-1}$ applied to mature seed Impatiens resulted in a 2- to 3 -fold increase in flower senescence $5 \mathrm{~d}$ after drench as compared with untreated plants (Mattson, unpublished data). Although flower and bud abscission was not observed when ethylenesensitive Impatiens plants were placed next to ethephon-drenched plants (Runkle, personal observation), it could be envisioned that when several thousand plants are drenched in a poorly ventilated greenhouse that nearby sensitive crops may be affected. More work is needed to assess any effect of ethephon drenches on neighboring crops, because ethylene in the air could accumulate to damaging levels for other crops.

\section{Conclusions}

This work demonstrates potential for ethephon application as a substrate drench for height control on a range of containerized floriculture crops. However, additional studies are necessary before ethephon drenches are adopted by the commercial industry. For example, we found that drench effects varied based on the specific crop and application rate and timing. More work is needed to determine any potential interactions with greenhouse environmental conditions and/or cultural practices. Further studies may help identify the mechanism of action for ethephon substrate drenches on stem extension. Finally, it should be noted that currently available ethephon products are not labeled for drench application.

\section{Literature Cited}

Anonymous. 1973. Narcissus pot plants, p. 83-86. In: Rosewarne Experimental Horticulture Station Annual Report. Camborne, UK.

Anonymous. 1974. Narcissus pot plants, p. 59-60. In: Rosewarne Experimental Horticulture Station Annual Report. Camborne, UK.

Barrett, J. 2004. Poinsettias: Early PGR drench? Greenhouse Product News 14:42-48.

Blanchard, M.G. and E.S. Runkle. 2007. Dipping bedding plant liners in paclobutrazol or uniconazole inhibits subsequent stem extension. HortTechnology 17:178-182.

Boldt, J.L. 2008. Whole plant response of chrysanthemum to paclobutrazol, chlormequat chloride, and (s)-abscisic acid as a function of exposure time using a splitroot system. MS thesis, Univ. of Florida, Gainesville, FL.

Briggs, J.E. 1975. The effects on growth and flowering of the chemical growth regulators ethephon on narcissus and ancymidol on tulip. Acta Hort. 47:287-296.

Currey, C.J., D.M. Camberato, A.P. Torres, and R.G. Lopez. 2010. Plant growth retardant drench efficacy is not affected by substrate containing parboiled rice hulls. HortTechnology 20:863-866.

Currey, C.J. and R.G. Lopez. 2011. Early flurprimidol drench applications suppress final height of four poinsettia cultivars. HortTechnology 21:35-40.

Davis, T.D., G.L. Steffens, and N. Sankhla. 1988. Triazole plant growth regulators. Hort. Rev. 10:63-105.

De Hertogh, A. 1996. Potted daffodils (Narcissus), p. B69-83. In: The Holland bulb forcer's guide. 5th Ed. Intl. Flower Bulb Ctr., Hillegom, The Netherlands.

Denne, M.P. 1960. Leaf development in Narcissus pseudonarcissus L. II. The comparative development of scale and foliage leaves. Ann. Bot. (Lond.) 24:32-47.

Dolan, L. 1997. The role of ethylene in the development of plant form. J. Exp. Bot. 48:201-210.

Edgerton, L.J. and A.H. Hatch. 1972. Absorption and metabolism of ${ }^{14} \mathrm{C}$ (2-chloroethyl) phosphonic acid in apples and cherries. J. Amer. Soc. Hort. Sci. 97:112-115.

Fisher, P.R. and R.D. Heins. 2002. UNH FloraTrack for poinsettia: Graphical tracking plant height on computer. Univ. New Hampshire Coop. Ext., Durham, NC.

Gent, M.P.N. and R.J. McAvoy. 2000. Plant growth retardants in ornamental horticulture, p. 89-146. In: Basara, A.S. (ed.). Plant growth regulators in agriculture and horticulture: Their role and commercial uses. Food Products Press, Binghamton, NY.

Glady, J.E., N.S. Lang, and E.S. Runkle. 2007. Effects of ethephon on stock plant management of Coreopsis verticillata, Dianthus caryophyllus, and Veronica longifolia. HortScience 42:16161621.

Johnson, C.R., D.B. McConnell, and J.N. Joiner. 1982. Influence of ethephon and light intensity on growth and acclimatization of Ficus benjamina. HortScience 17:614-615.
Krug, B.A., B.E. Whipker, I. McCall, and J.M. Dole. 2005. Comparison of flurprimidol to ethephon, paclobutrazol and unicolazole for hyacinth height control. HortTechnology 15: 872-874.

Krug, B.A., B.E. Whipker, I. McCall, and J.M. Dole. 2006a. Narcissus response to plant growth regulators. HortTechnology 16:129-132.

Krug, B.A., B.E. Whipker, and I. McCall. 2006b. Hyacinth height control using preplant bulb soaks of flurprimidol. HortTechnology 16:370 375.

Leatherwood, R.W., J.M. Dole, and J.E. Faust. 2009. Ethephon residual catalysis on unrooted Impatiens hawkeri cuttings and stock plants. HortScience 44:532-535.

Lopez, R.G. and E.S. Runkle. 2007. Early PGR drench applications on poinsettia. Greenhouse Product News 19(8):22-29.

Martin, G.C., H.A. Abdel-Gawad, and R.J. Weaver. 1972. The movement and fate of (2-chloroethyl) phosphonic acid in walnut. J. Amer. Soc. Hort. Sci. 97:51-54.

Maynard, J.A. and J.M. Swan. 1963. Organophosphorus compounds I. 2-chloroalkylphosphonaiccids as phosphorylating agents. Aust. J. Chem. 16:596-608.

Miller, W.B. 1992. Easter and hybrid lily production. Timber Press, Portland, OR

Miller, W.B. 1993. Lilium longiflorum, p. 391-422. In: De Hertogh, A. and M. LeNard (eds.). The physiology of flowering bulbs. Elsevier, Amsterdam, The Netherlands.

Miller, W.B. 2011. Website for the Flower Bulb Research Program. Cornell Univ., Ithaca, NY. 9 June 2011. <http://www.flowerbulbs.cornell.edu>

Moe, R. 1980. The use of ethephon for control of plant height in daffodils and tulips. Acta Hort. 109:197-204.

Rademacher, W. 2000. Growth retardants: Effects on gibberellin biosynthesis and other metabolic pathways. Ann. Rev. Plant Physiol. Plant Mol. Biol. 51:501-531.

Reid, M.S. 1987. Ethylene in plant growth, development, and senescence, p. 257-279. In: Davies, P.J. (ed.). Plant hormones and their role in plant growth and development. Martinus Nijhoff, Boston, MA.

Runkle, E.S., C.M. Whitman, and M. Olrich. 2006. Determining effects of a uniconazole drench on Celosia, Petunia, Salvia, and Tagetes. HortScience 41:1067 (abstr.).

Starman, T.W., M.C. Robinson, and K. Eixmann. 2004. Efficacy of ethephon on vegetative annuals. HortTechology 14:83-87.

Styer, R.C. 2002. Using Florel effectively. Greenhouse Product News 12:10-15.

Thompsett, A.A. 1974. Bulbs, p. 16-86. In: Rosewarne Experimental Horticulture Station Annual Report. Camborne, UK.

U.S. Department of Agriculture. 2011. Floriculture crops 2010 summary. Nat. Agr. Sta. Service, Washington, DC.

Whipker, B.E. and I. McCall. 2000. Response of potted sunflower cultivars to daminozide foliar sprays and paclobutrazol drenches. HortTechnology 10:209-211. 